\title{
Mortalidad quirúrgica durante pandemia de coronavirus en un centro universitario
}

\author{
Carlos Ignacio Domínguez C. ${ }^{1}$, Ricardo Gallardo G. ${ }^{1}$, Mariluna Corvalán G. ${ }^{1}$, \\ Rodrigo Sánchez M. ${ }^{1}$, Gabriel Sandoval S. ${ }^{1}$ y Jaime Rappoport S. ${ }^{1}$
}

\section{Surgical mortality during a coronavirus pandemic in a university center}

Background: The coronavirus pandemic, started in the city of Wuhan in 2019, has disrupted the world and deeply affected surgical activity. Restricting the number of interventions dramatically, after initial reports of postoperative mortality over $20 \%$ in patients with COVID. The purpose of this study is to evaluate the figures for surgical mortality, during the coronavirus pandemic. Materials and Method: Retrospective cohort of patients operated between March 15, 2020 and July 31, 2020 at a university center. Clinical variables associated with surgical intervention and coinfection by SARS-CoV-2 were evaluated. Results: 344 patients with an overall mortality of $6.1 \%$ were analyzed. PCR testing for COVID was performed on 153 patients. Only from the ninth week of the pandemic did routine preoperative testing begin. Patients who presented a higher risk of mortality were: PCR $\operatorname{COVID}^{(+)}(22.7 \%)$, arterial hypertension $(11.6 \%)$ and age over 60 years $(12.4 \%)$. In the present series, the following variables were not statistically significant risk factors for mortality: gender, obesity, diabetes mellitus, oncological pathology, emergency surgery and ASA classification. When analyzing two subgroups, we observed that COVID negative patients under 60 had a mortality rate of $1.26 \%$, versus $36.36 \%$ in those over 60 years of age, COVID positive. Discussion: The results of the present study lead us to make every effort to rule out COVID infection preoperatively to reduce the risks of postoperative mortality. Although this is a series of cases and the extrapolation of its results should be cautious, having national figures can be a useful element to make decisions in this stage of reactivation of surgical activity.

Keywords: Coronavirus; pandemic; COVID-19; surgery; postoperative mortality.

\section{Resumen}

Introducción: La pandemia de coronavirus, iniciada en Wuhan el año 2019, ha trastocado al mundo y afectado profundamente a la actividad quirúrgica al restringir el número de intervenciones en forma dramática, después de los reportes iniciales de mortalidad posoperatoria sobre el $20 \%$ en pacientes operados portadores de COVID. El objetivo del presente estudio fue evaluar las cifras de mortalidad quirúrgica, en pacientes intervenidos quirúrgicamente durante la pandemia del COVID-19. Materiales y Métodos: Cohorte retrospectiva de pacientes operados entre el 15 de marzo de 2020 y el 31 de julio de 2020 en un centro universitario. Se evaluó variables clínicas asociadas a la intervención quirúrgica y coinfección por SARS-CoV-2. Resultados: Se analizaron 344 pacientes quienes presentaron una mortalidad global de 6,1\%. Se realizó examen de PCR para COVID a 153 pacientes. Presentaron un riesgo de mortalidad significativo los pacientes: PCR COVID ${ }^{(+)}(22,7 \%), p=0,01$, portadores de hipertensión arterial $(11,6 \%)$ $\mathrm{p}=0,03$ y mayores de 60 años $(12,4 \%) \mathrm{p}<0,001$. No fueron factores estadísticamente significativos de mayor riesgo de mortalidad, las siguientes variables: género, obesidad, diabetes mellitus, patología oncológica, cirugía de urgencia y clasificación de ASA. Al analizar dos subgrupos se observó que los pacientes menores de 60 años COVID negativo presentaron una cifra de mortalidad de 1,26\% versus 36,3\% en los mayores de 60 años, COVID positivos $(\mathrm{p}=0,01)$. Discusión: Los resultados del presente estudio sugieren que se deben realizar los mayores esfuerzos para descartar la infección por SARS-CoV-2 en la evaluación preoperatoria para disminuir los riesgos de mortalidad posoperatoria.

Palabras clave: Coronavirus; pandemia; COVID-19; cirugía; mortalidad posoperatoria.
Departamento de Cirugía Hospital Clínico Universidad de Chile, Santiago, Chile.

Aprobado por el Comité de Ética local.

Recepción 2020-12-21 aceptado 2021-03-22

Correspondencia a: Dr. Carlos Ignacio Domínguez C. carlosdominguez@uchile.cl 


\section{Introducción}

La pandemia de coronavirus, iniciada en la ciudad de Wuhan en 2019 , ha trastocado al mundo y afectado profundamente a la actividad quirúrgica.

El reporte inicial de Lei $^{1}$, con una mortalidad de 20,5\% (7/34) en pacientes portadores de COVID-19, en Wuhan, trajo como consecuencia que se paralizara la actividad quirúrgica electiva en el país, siguiendo las recomendaciones de diversas sociedades nacionales e internacionales ${ }^{2-5}$, y las normativas del Ministerio de Salud (MINSAL) chileno, por resolución del 25 de marzo de $2020^{6}$.

La alerta de una elevada letalidad en cirugía, en pacientes COVID-19 positivos, fue ratificada en el estudio cooperativo CovidSurg Collaborative Group, que reportó una cifra de $23,8 \%$ de mortalidad en 1.128 pacientes de diversos países?

En nuestro centro, asistimos a una reducción de un $90 \%$ de la actividad quirúrgica después de estas recomendaciones. Sin embargo, se mantuvieron algunas cirugías oncológicas, en las cuales su postergación por más de tres meses significaba una agravación del pronóstico, así como las cirugías de urgencia y emergencia y las denominadas tiempos sensibles.

El propósito del presente estudio fue evaluar la mortalidad a 30 días de una serie de pacientes intervenidos bajo estos criterios, en nuestro centro para tener una referencia del riesgo de efectuar cirugía en estas condiciones. El objetivo primario fue determinar la mortalidad operatoria. El objetivo secundario fue analizar los factores de riesgo para esta mortalidad.

\section{Material y Método}

Se efectuó un estudio observacional, basado en una cohorte retrospectiva de pacientes operados en el Departamento de Cirugía del Hospital Clínico de la Universidad de Chile, durante la pandemia de coronavirus (marzo a julio de 2020).

Los criterios de inclusión fueron todos los pacientes operados entre el 15 de marzo y 31 de julio de 2020. Se excluyeron pacientes operados por otros departamentos quirúrgicos, tales como Ginecología, Otorrinolaringología, Oftalmología, Traumatología y Neurocirugía.

Se realizó una revisión sistemática del registro clínico y seguimiento telefónico de todos los pacientes intervenidos, registrando datos epidemiológicos y clínicos asociados a la intervención quirúrgica y coinfección por SARS-CoV-2. El seguimiento telefónico se realizó a todos los pacientes dados de alta vivos, cumplidos al menos 30 días desde el egreso hospitalario. Se consideró paciente COVID $(+)$ solo a aquellos pacientes con examen de PCR para SARS-CoV-2 Positivos, realizado por un laboratorio validado por MINSAL. Se definió como obesidad a los pacientes con IMC $\geq 30 \mathrm{~kg} / \mathrm{m}^{2}$.

Las variables estudiadas fueron: edad, género, IMC (índice de masa corporal en $\mathrm{kg} / \mathrm{m}^{2}$ ), diagnóstico de la patología de base, intervención quirúrgica efectuada, fecha, ASA (riesgo anestésico de la Sociedad Americana de Anestesia), criterio de cirugía electiva o de urgencia, mortalidad a 30 días y la presencia de comorbilidades como: diabetes, hipertensión arterial (HTA), patología respiratoria u otra.

Posterior a la recolección de información, se realizó un análisis estadístico utilizando una base de datos anonimizada, para resguardar la identidad de los pacientes. Se utilizó software estadístico (STATA v13) y se efectuó un análisis descriptivo de variables continuas con medidas de resumen y de variables discontinuas con medidas de frecuencia, utilizando test paramétricos y no paramétricos dependiendo de la distribución de las variables, para contrastar diferencias entre los grupos. Se utilizó una regresión logística múltiple para determinar el impacto de las variables estudiadas en la mortalidad. Se consideró un nivel de significación estadístico $\mathrm{p}<0,05$.

No se establece tamaño muestral, dado que se evaluaron a todos los pacientes intervenidos durante el periodo de estudio.

\section{Resultados}

La presente serie comprende 344 pacientes, con una edad promedio $54,2 \pm 18,5$ años, La mayor frecuencia se observó entre los 30 y 60 años $161 / 344=46,8 \%$ (Tabla 1 y Figura 1 ).

El 54,07\% (186) correspondió a pacientes ingresados en forma electiva para resolución quirúrgica, siendo las patologías más frecuentes las de origen vascular, digestivo y coloproctológicas (Tabla 2).

De los pacientes intervenidos 174 (50,58\%) correspondió al género femenino (Tabla 3 ).

El IMC promedio fue de $27,12 \pm 5,45 \mathrm{~kg} / \mathrm{m}^{2}$, se presentó obesidad (IMC > 30) en $228(68,2 \%)$, pacientes (Tabla 3 ).

Al evaluar la clasificación de riesgo anestésico según ASA, se observa una mayor frecuencia de pacientes ASA II, 201 (58,43\%) (Tabla 3$).$ 
Tabla 1. Distribución de pacientes según edad, agrupados por décadas y mortalidad quirúrgica

\begin{tabular}{|cccc|}
\hline Décadas & Vivos & Fallecidos & Total \\
\hline $15-19$ & 17 & 0 & 17 \\
$20-29$ & 25 & 0 & 25 \\
$30-39$ & 40 & 0 & 40 \\
$40-49$ & 44 & 0 & 44 \\
\hline $50-59$ & 70 & 2 & 72 \\
$60-69$ & 64 & 7 & 71 \\
$70-79$ & 42 & 4 & 46 \\
$80-89$ & 21 & 6 & 27 \\
$90-99$ & 0 & 2 & 2 \\
Total & 323 & 21 & 344 \\
\hline
\end{tabular}

Las comorbilidades analizadas fueron: hipertensión arterial, presente en 112 pacientes $(32,56 \%)$, diabetes mellitus en $76(22,09 \%)$ y patología oncológica en 68 pacientes $(19,76 \%)$ (Tabla 3$)$.

La mortalidad global de la presente serie fue de $21 / 344=6,1 \%$. La mortalidad de los pacientes en quienes se estudió la PCR fue de 13/153 = 8,49\%. En los casos PCR positivos se observó una mortalidad del $22,73 \%$ y en los casos PCR negativo 6,1\% (8/131), p 0,01 (Tabla 4). En los pacientes dados de alta, el seguimiento telefónico a 30 días, no reveló mortalidad posterior al alta.

El análisis univariable, evidenció una asociación entre mortalidad con HTA $(\mathrm{p}=0,03)$, COVID $(+)$ $(p=0,01)$ y grupo etario $(p<0,001)$, no observándose asociación significativa con diabetes mellitus, cirugía de urgencia, patología oncológica, clasificación ASA, obesidad ni el género (Tabla 3).

La regresión logística multivariable, mostró que la edad presentó un Odds Ratio (OR) de 1,10, con un intervalo de confianza (IC) de 1,03-1,19; la presencia de hipertensión arterial (OR 7,18; IC 1,03-50,27) y el resultado de PCR COVID positivo, (OR 7,19; IC 1,17-44,10), representaron un significativo aumento del riesgo de mortalidad (Tabla 5).

En los 21 pacientes fallecidos, se detallan las variables estudiadas en la Tabla 6. Patología oncológica se registró en 12 , cuadros sépticos en 8 , progresión de la enfermedad de base en 13 y dos pacientes presentaron neumonía COVID, factores relevantes en la evolución.

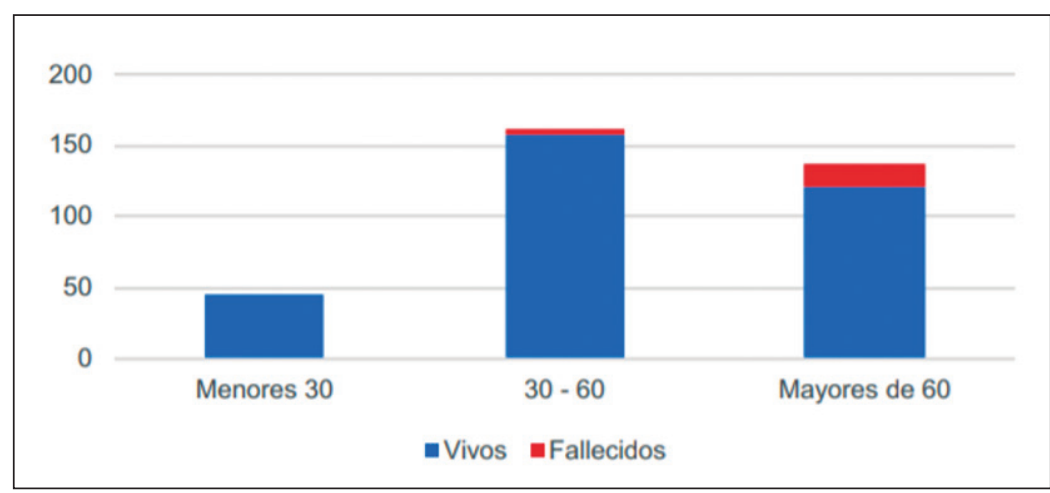

Figura 1. Distribución de mortalidad por grupo etarios.

Tabla 2. Distribución de pacientes de acuerdo a especialidad, tipo de cirugía (electiva/urgencia) y mortalidad

\begin{tabular}{|lcccccc|}
\hline Equipo & Urgencia & Electivo & Total & Fallecen & \% & p \\
Vascular & 53 & 40 & 93 & 4 & 9,7 & 0,195 \\
Coloproctología & 48 & 34 & 82 & 4 & 4,9 & \\
Digestivo & 46 & 43 & 89 & 6 & 3,4 & \\
Endocrino & 10 & 14 & 24 & 2 & 4,2 & \\
Hernia & 8 & 9 & 17 & 2 & 17,6 & \\
Tórax & 13 & 13 & 26 & 2 & 3,8 & \\
Plástica & 8 & 5 & 13 & 0 & 0 & \\
Total & 186 & 158 & 344 & 21 & 6,1 & \\
\hline
\end{tabular}

Tabla 3. Análisis univariado de variables estudiadas

\begin{tabular}{|c|c|c|c|c|c|c|c|c|}
\hline \multirow{2}{*}{\multicolumn{2}{|c|}{ Variables }} & \multicolumn{2}{|c|}{ General } & \multicolumn{2}{|c|}{ Vivos } & \multicolumn{2}{|c|}{ Muertos } & \multirow[t]{2}{*}{ p } \\
\hline & & n & $\%$ & $\mathbf{n}$ & $\%$ & $\mathbf{n}$ & $\%$ & \\
\hline \multicolumn{2}{|c|}{ PCR COVID (+) } & 22 & 14,4 & 17 & 77,8 & 5 & 22,7 & 0,01 \\
\hline \multicolumn{2}{|l|}{ HTA } & 112 & 32,6 & 99 & 88,4 & 13 & 11,6 & 0,03 \\
\hline \multicolumn{2}{|c|}{ Obesidad } & 228 & 68,2 & 214 & 93,9 & 14 & 6,1 & 0,904 \\
\hline \multicolumn{2}{|c|}{ Diabetes mellitus } & 76 & 22,1 & 71 & 93,4 & 5 & 6,6 & 0,845 \\
\hline \multicolumn{2}{|c|}{ Patología oncológica } & 68 & 19,8 & 62 & 91,2 & 6 & 8,8 & 0,296 \\
\hline \multicolumn{2}{|c|}{ Cirugía de urgencia } & 158 & 45,9 & 148 & 93,7 & 10 & 6,3 & 0,873 \\
\hline \multirow[t]{4}{*}{ ASA } & I & 51 & 14,8 & 46 & 90,2 & 5 & 9,8 & 0,652 \\
\hline & II & 201 & 58,4 & 190 & 94,5 & 11 & 5,5 & \\
\hline & III & 80 & 23,3 & 76 & 95,0 & 4 & 5,0 & \\
\hline & IV & 12 & 34,9 & 11 & 91,7 & 1 & 8,3 & \\
\hline \multirow[t]{3}{*}{ Edad } & $<30$ & 46 & 13,4 & 46 & 100 & 0 & 0 & $<0,001$ \\
\hline & $30-60$ & 161 & 46,8 & 157 & 97,5 & 4 & 2,5 & \\
\hline & $>60$ & 137 & 39,8 & 120 & 87,6 & 17 & 12,4 & \\
\hline \multicolumn{2}{|c|}{ Femenino } & 174 & 50,6 & 163 & 93,7 & 11 & 6,3 & 0,865 \\
\hline
\end{tabular}


Tabla 4. Mortalidad vs. PCR

\begin{tabular}{|lccc|}
\hline PCR COVID & Vivos & Fallecidos & Total \\
\hline Positivo & 17 & 5 & 22 \\
Negativo & $(77,27 \%)$ & $(22,73 \%)$ & 131 \\
No realizada & 123 & 8 & 191 \\
& $(93,89 \%)$ & $(6,11 \%)$ & \\
Total & 183 & 8 & 344 \\
& $(95,81 \%)$ & $(4,19 \%)$ & \\
\hline
\end{tabular}

$\chi^{2}$ al comparar distribución de grupo $\mathrm{p}=0,003$.
Tabla 5. Análisis multivariado de mortalidad quirúrgica $(n=153$ pacientes $)$

\begin{tabular}{|lll|}
\hline Variables & OR & IC \\
Edad & 1,1 & $1,03-1,19$ \\
IMC & 0,93 & $0,80-1,09$ \\
PCR COVID (+) & 7,19 & $1,17-44,10$ \\
HTA & 7,18 & $1,03-50,27$ \\
Diabetes mellitus & 0,26 & $0,04-1,75$ \\
Patología oncológica & 5,33 & $0,61-46,20$ \\
Cirugía de urgencia & 1,81 & $0,30-10,96$ \\
ASA & 0,46 & $0,13-1,60$ \\
Sexo masculino & 0,88 & $0,19-3,96$ \\
\hline
\end{tabular}

Tabla 6. Pacientes fallecidos

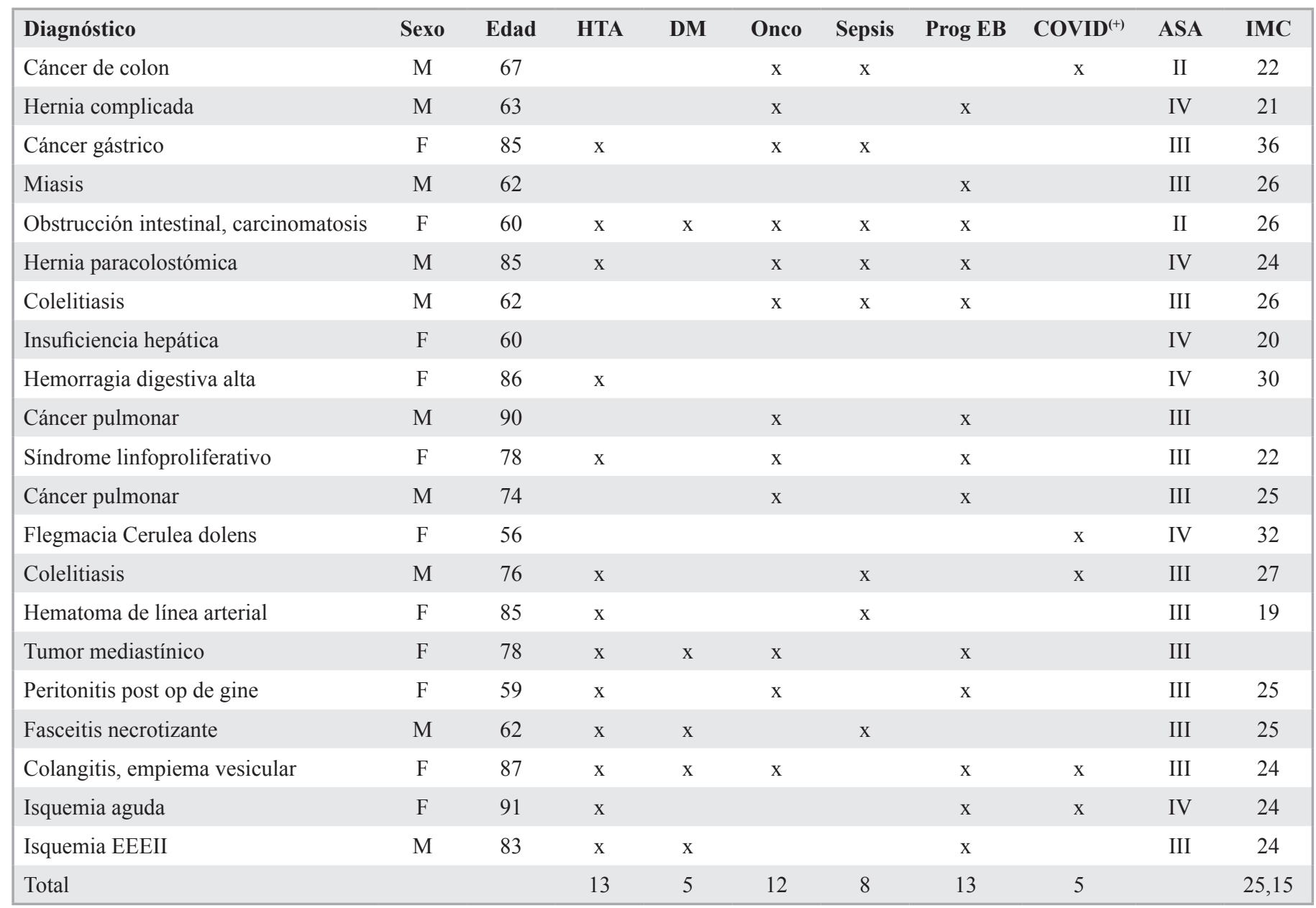

M: masculino; F: memenino; HTA: hipertensión arterial; DM: diabetes mellitus; ONCO: oncológico; PROG EB: progresión de enfermedad de Base; COVID ${ }^{(+)}$: infección por SARS-CoV-2 confirmada por estudio PCR; ASA: clasificación de riesgo de pacientes según American Society of Anesthesiologist; IMC: índice de masa corporal $\left(\mathrm{kg} / \mathrm{m}^{2}\right)$. 


\section{Discusión}

La mortalidad global de la presente serie, que fue de $6,1 \%$, está dada en un contexto de pandemia de coronavirus y refleja la selección de pacientes que se operaron, que fueron las cirugías catalogadas de urgencia y emergencia, las oncológicas no diferibles y pacientes sintomáticos con alto riesgo de desarrollar complicaciones de su patología de base. La mortalidad reportada en condiciones habituales para estas patologías es menor en rangos de $1 \%$ a $7 \% \%^{8-16}$.

El análisis univariable demostró que el hecho de tener una PCR $(+)$ y ser sometido a una cirugía, se asocia a un riesgo de mortalidad de $5 / 22=22,72 \%$ con un OR de 7,19 (IC 1,17-44,10) (Tablas 4 y 5). Cifra similar a la reportada por $\mathrm{Lei}^{1}$, de un $20,5 \%$ en 34 pacientes de la ciudad de Wuhan, al inicio de la pandemia y el $23,8 \%$ del estudio multinacional del CovidSurg Collaborative Group ${ }^{7}$ en 1.128 pacientes y representa el riesgo de mortalidad de los pacientes COVID positivos, operados en tiempos de pandemia.

Analizando las cifras de mortalidad en mayores y menores de 60 años, según PCR positiva o negativa, se observó un rango de mortalidad desde un 1,26\% en los menores de 60 años con PCR negativa hasta un 36,36\% en los mayores de 60 años PCR positiva (Figura 2), diferencia estadísticamente significativa $(\mathrm{p}<0,001)$.

Este elevado riesgo de mortalidad, obliga a realizar los mayores esfuerzos para diagnosticar a los pacientes COVID positivos en el preoperatorio, y restringir la cirugía en estos pacientes solo a las emergencias. No existe aún un consenso definitivo acerca del mejor momento para operar un paciente que presentó la enfermedad de coronavirus y cuánto tiempo se debe esperar después de haberse recuperado.

La presencia de comorbilidades como diabetes mellitus, hipertensión arterial, edad avanzada, obesidad y patología oncológica, entre otras, han sido reportadas por diversos autores, como factores de riesgo de mayor mortalidad posoperatoria. En la presente serie se observó que la diabetes mellitus se presentó en $76 / 344(22,09 \%)$ y se asoció a un riesgo de mortalidad de $5 / 76=6,57 \%$ versus los no diabéticos de 5,9\% (16/268) con un OR de 0,26 (IC $0,04-1,75)$ y $\mathrm{p}=0,845$ (Tablas 3 y 5 ). Estas cifras no concuerdan con lo reportado por Zhou ${ }^{17}$, Muniyappa $^{18}$ y Apicella ${ }^{19}$ que recalcan el mayor riesgo de mortalidad de los pacientes diabéticos operados en pandemia.

La presencia de hipertensión arterial en la presente serie representó un significativo mayor riesgo de

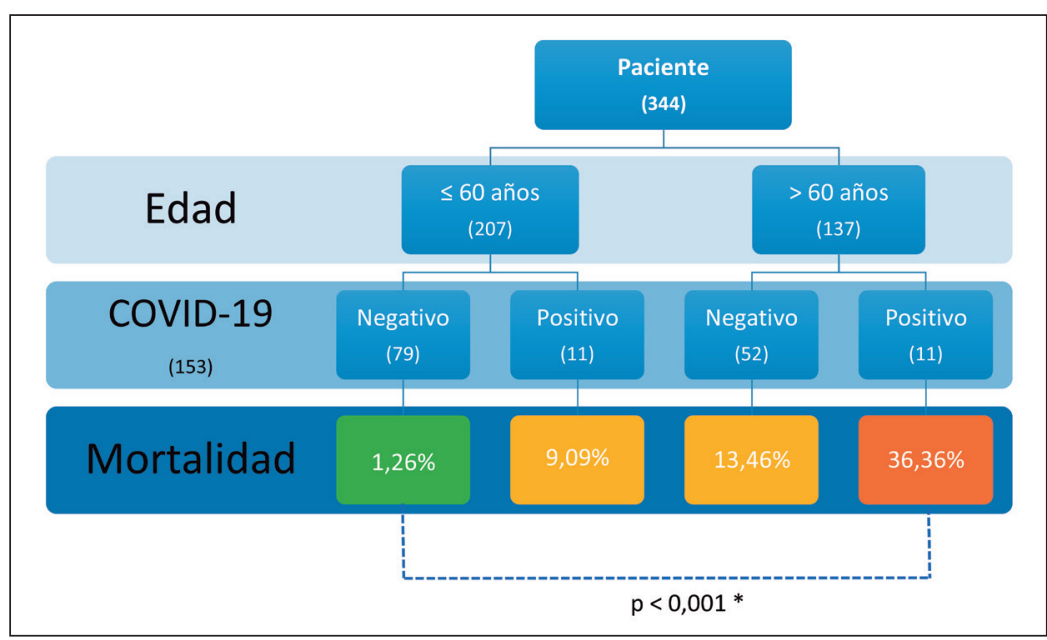

Figura 2. Mortalidad según edad y COVID-19. Comparación entre grupo $\leq 60$ años COVID $(-)$ y $>60$ años COVID (+) utilizando test de Chi 2 con $p<0,001$.

mortalidad, con un OR de 7,18 (IC 1,03-50,27), lo que concuerda con lo reportado por Fang ${ }^{20}$ (Tabla $5)$.

Los pacientes portadores de patología oncológica presentaron un riesgo de mortalidad de $8,82 \%$ versus $5,34 \%$, del resto de las patologías, con un OR de 5,33 (IC 0,61-46,20) (Tablas 3 y 5), sin alcanzar significación estadística. Este aumento del riesgo podría explicarse por algún grado de inmunodeficiencia de los pacientes portadores de patología oncológica. Al-Shamsi ${ }^{21}$, postula un riesgo aumentado al doble de contraer COVID, en los pacientes portadores de neoplasias, por inmunodeficiencia y tratamientos complementarios, como radio y quimioterapia. Por otra parte, Onder $^{22}$ señala que el $24,5 \%$ de una subpoblación de pacientes fallecidos en Italia, eran portadores de cánceres activos.

La obesidad, no se asoció con mayor riesgo de mortalidad (Tabla 3), a diferencia de lo reportado por diversos autores como $\mathrm{Zhou}^{17}$ y Yang $^{23}$. El IMC promedio de la presente serie fue de 27,12.

En este estudio se documentó un aumento de la mortalidad paralelo al aumento de la edad de los pacientes, especialmente en los mayores de 60 años, (Tablas 1 y 7, Figura 1). Esto concuerda con lo reportado por diversos autores, como Onder $^{22}$ y Yang $^{24} \mathrm{y}$ podría estar en contexto de mayores patologías asociadas.

La evaluación del ASA no mostró en la presente serie diferencias de riesgo estadísticamente significativa, al igual que la cirugía de urgencia versus la electiva (Tabla 5).

En el análisis multivariable, se documentó un significativo aumento de la mortalidad con la coe- 
xistencia de hipertensión arterial, $\operatorname{COVID}^{(+)}$y edad mayor de 60 años (Tabla 5).

El examen de PCR preoperatorio de rutina se inició en el curso de la novena semana de pandemia.

Por las condiciones epidemiológicas del país en las fases iniciales de la pandemia, se podría asumir que la gran mayoría del resto de los pacientes deben de haber sido negativos, lo que daría una mortalidad global en los pacientes COVID negativo de $16 / 322=4,96 \%$.

En la etapa actual de reactivación del quehacer quirúrgico, esta cifra de riesgo global está muy distante de las elevadas cifras reportadas por Lei ${ }^{1}$ y CovidSurg Collaborative Group ${ }^{7}$, lo que avalaría el ampliar el quehacer quirúrgico, en esta etapa de la pandemia, para evitar los negativos efectos de la postergación de los pacientes candidatos a cirugía programada.

En condiciones epidemiológicas de bajo riesgo, con menos de 10 casos activos por 100.000 habitantes, las medidas de triage preoperatorio, con encuesta $48 \mathrm{~h}$ antes de la cirugía y al momento del ingreso, PCR negativo $48 \mathrm{~h}$ antes del ingreso y cuarentena por 14 días preoperatorio, hacen que el riesgo de operar un paciente falso negativo, o en periodo de incubación, podría estimarse en $1 / 50.000^{25}$.

Los resultados del presente estudio sugieren que no se debe seguir postergando indefinidamente las cirugías oncológicas, tiempos sensibles y las que en condiciones electivas y pacientes COVID tienen bajo riesgo, como la cirugía biliar y la patología herniaria. Cuando llegan de urgencia, estas representan un mayor riesgo y se asocian a mayores costos de atención, lo que, en un sistema de salud tensionado como el actual, no es un tema menor. Sin embargo, estos resultados no deben ser extrapolados a otras situaciones y merecen ser analizados con cautela en el momento de decidir una cirugía que no es imprescindible mientras dure la pandemia.

La pandemia de coronavirus ha planteado múltiples interrogantes, para las cuales aún no tenemos respuestas con certezas. Es imprescindible contar con otras cifras nacionales de riesgo de mortalidad quirúrgica en época de pandemia, para tomar la mejor decisión frente a cada paciente en particular.

En conclusión, los resultados del presente estudio referente a los factores de riesgo en pacientes chilenos sometidos a tratamiento quirúrgico en época de pandemia por COVID-19, muestran que la edad, la presencia de hipertensión arterial y el resultado de test de COVID positivo representan un significativo alto riesgo de mortalidad posoperatoria.

\section{Agradecimientos}

A todos nuestros colegas y personal de colaboración, que constituyeron la primera línea de tratamiento durante la primera ola de la pandemia y fueron los tratantes de estos pacientes. Al Dr. Attila Csendes J. por su apoyo y colaboración para realizar este trabajo.

\section{Responsabilidades éticas}

Protección de personas y animales. Los autores declaran que para esta investigación no se han realizado experimentos en seres humanos ni en animales.

Confidencialidad de los datos. Los autores declaran que en este artículo no aparecen datos de pacientes.

Conflictos de interés: no hay.

\section{Bibliografía}

1. Lei S, Jiang F, Su W, Chen C, Chen J, Mei W, et al. Clinical characteristics and outcomes of patients undergoing surgeries during the incubation period of COVID-19 infection. EClinicalMedicine 2020;21:100331. doi: 10.1016/j. eclinm.2020.100331. eCollection 2020 Apr.

2. Sociedad de Cirujanos de Chile: Recomendaciones de la Sociedad de Cirujanos de Chile, para la organización de Servicios de Cirugía, en zonas de alta y baja afectación por COVID-19. [30-10-
2020]. Disponible en https://www.socich. $\mathrm{cl} /$ recomendaciones-en-al-pandemia-delcovid-19/.

3. American College of Surgeons: Resources for the surgical community, COVID-19 an Surgery. Disponible en https://www.facs.org/covid-19/clinicalguidance.

4. Society of American Gastrointestinal and Endoscopic Surgeons: SAGES and EAES recomendendations regarding surgical responses to COVID-19 crisis. Disponible en https://www.sages.org/ recommendations-surgical-responsecovid-19/.
5. Dabanch J. Cirugía en tiempos de COVID. Rev Chil Cir 2020;72:381-3.

6. Ministerio de Salud, Chile: Dispone medidas sanitarias que indica por brote de COVID-19. Resolución Núm. 208 exenta, 2020. Disponible en https://www.minsal. cl/wp-content/uploads/2020/03/1745861 web.pdf.

7. COVIDSurg Collaborative. Mortality and pulmonary complications in patients undergoing surgery with perioperative SARS-CoV-2 infection: an international cohort study. Lancet 2020;396:27-38.

8. Csendes A, Braghetto I, Díaz JC, Castillo J, Rojas J, Cortés S. Morbilidad y 
mortalidad operatoria de la gastrectomía subtotal y total por cáncer gástrico 2004 a 2010: Parte I de un estudio prospectivo. Rev Chil Cir. 2011;63:585-90.

9. Kim HH, Hyung WJ, Cho GS, Kim MC, Han SU, Kim W, et al. Morbidity and mortality of laparoscopic gastrectomy versus open gastrectomy for gastric cancer: an interim report-a phase III multicenter, prospective, randomized Trial (KLASS Trial). Ann Surg. 2010;251:41720. doi: 10.1097/SLA.0b013e3181cc8f6b.

10. Bachmann J, Michalski CW, Martignoni ME, Büchler MW, Friess H. Pancreatic resection for pancreatic cancer. HPB (Oxford). 2006;8:346-51. doi: 10.1080/13651820600803981.

11. Bannura C, Cumsille M, Barrera A, Contreras J, Melo C, Soto D. Resultados del tratamiento quirúrgico del cáncer colorrectal: Análisis de la recurrencia y sobrevida en 400 pacientes. Rev Med Chile 1995;123:464-72.

12. Alustizaa J, Cermeño B, Campo E, Romeo JA. Sardón JD, Reka L, et al. Factores de riesgo de mortalidad postoperatoria en el cáncer colorrectal: seguimiento de una cohorte en una unidad especializada. Cir Esp. 2010;87:101-7.

13. Ibáñez L, Escalona A, Devaud N, Montero P, Ramírez E, Pimentel F, et al. Colecistectomía laparoscópica: experiencia de 10 años en la Pontificia
Universidad Católica de Chile. Rev Chil Cir. 2007;59:10-5.

14. Csendes A, Yarmuch J, Díaz JC, Castillo J Maluenda F. Causas de mortalidad por colecistectomía tradicional y laparoscópica 1991-2010. Rev Chil Cir. 2012;64:555-9.

15. Cárcamo C, Rivera E, Schwaner A. Apendicitis aguda en el Hospital Clínico Regional de Valdivia. Cuad. Cir. 2002;16:11-5.

16. Devereaux PJ, Sessler D. Cardiac Complications in Patients Undergoing Major Noncardiac Surgery. N Engl J Med. 2015;373:2258-69.

17. Zhou Y, Chi J, Lv W, Wang Y. Obesity and diabetes as high-risk factors for severe coronavirus disease 2019 (COVID-19). Diabetes Metab Res Rev. 2021;37(2):e3377. doi: 10.1002/ dmrr.3377. Epub 2020 Jul 20.

18. Muniyappa R, Gubbi S. COVID-19 pandemic, coronaviruses, and diabetes mellitus. Am J Physiol Endocrinol Metab. 2020;318: E736-E741.

19. Apicella M, Campopiano MC, Mantuano M, Mazoni L, Coppelli A, Del Prato S. COVID-19 in people with diabetes: understanding the reasons for worse outcomes. The Lancet Diabetes \& Endocrinology 2020;8:782-92.

20. Fang L, Karakiulakis G, Roth M. Are patients with hypertension and diabetes mellitus at increased risk for COVID-19 infection? Lancet Respir Med. 2020;8:E21. doi: 10.1016/S22132600(20)30116-8.

21 Al-Shamsi HO, Alhazzani W, Alhuraiji A, Coomes EA, Chemaly RF, Almuhanna $M$, et al. A Practical Approach to the Management of Cancer Patients During the Novel Coronavirus Disease 2019 (COVID-19) Pandemic: An International Collaborative Group. Oncologist 2020; 25:e936-e945.

22. Onder G, Rezza G, Brusaferro S. Casefatality rate and characteristics of patients dying in relation to COVID-19 in Italy. Jama 2020;323:1775-6.

23. Yang J, Zheng Y, Gou X, Pu K, Chen Z, Guo Q, et al. Prevalence of comorbidities and its effects in patients infected with SARS-CoV: a systematic review and meta-analysis. International Journal of Infectious Diseases 2020;94:91-5.

24. Yang X, Yu Y, Xu J, Shu H, Xia J, Liu H, et al. Clinical course and outcomes of critically ill patients with SARS-CoV-2 pneumonia in Wuhan, China: a single-centered, retrospective, observational study. Lancet Respir Med. 2020;8:475-81.

25. Danilla S. New algorithm for safety in elective surgery in the COVID era statistics and evidence. Latam Safety Task Force. Webinar 9 mayo 2020. 